\title{
Ist Forschung zur Komplementärmedizin Zeit- und Geldverschwendung?
}

\author{
Klaus Linde \\ Zentrum für naturheilkundliche Forschung, Technische Universität München, Deutschland
}

Das New England Journal of Medicine und der Lancet gelten nach wie vor als die wichtigsten Journals für die klinische Medizin. Veröffentlichungen zu komplementärmedizinischen Themen sind darin äusserst selten. Umso höher ist der Signalwert von zwei Editorials einzuschätzen, die kürzlich dort erschienen sind. Im New England Journal of Medicine vom 28. Juli 2005 kommentierte der bekannte Skeptiker Wallace Sampson [1] eine im selben Heft publizierte, negative Echinaceastudie [2]. Am Ende seines Editorials kritisiert Sampson massiv, dass in den USA über das National Center of Complementary and Alternative Medicine (NCCAM) grosse Summen öffentlicher Gelder für klinische Forschung zu unplausiblen Therapien ausgegeben werden (siehe hierzu auch seine Ausführungen unter www.quackwatch.org/01QuackeryRelatedTopics/nccam.html). Die Hinwendung zur «alternativen Medizin» wird als Teil unsinniger gesellschaftlicher Trends («errant social-medical trends») bezeichnet, deren Erforschung letztlich Ressourcenverschwendung sei. Sampson greift das Wort des schillernden Skeptikers Petr Skrabanek von der «demarcation of the absurd» auf, die aus seiner Sicht bei der komplementärmedizinischen Forschung vernachlässigt wird.

Am 27. August 2005 erschien dann im Lancet eine grosse Metaanalyse der placebokontrollierten Studien zur Homöopathie [3], die im Gegensatz zu der von unserer Arbeitsgruppe 1997 vorgelegten [4] zu deutlich negativeren Ergebnissen kommt. Das Herausgeberteam des Lancet schreibt im begleitenden Editorial unter dem Titel «The End of Homoeopathy» von «absurd dilutions», «surely the time has passed for ... further investment in research ...» und «now doctors have to be bold and honest with their patients about homoeopathy's lack of benefit ...» [5].

Bis vor etwa 30 Jahren liess sich mit dem Argument der mangelnden wissenschaftlichen Plausibilität die Forschung zu wirklich umstrittenen Verfahren weitgehend verhindern. Mit dem Aufkommen von Strömungen wie evidenzbasierter Medizin, Versorgungsforschung oder Outcomes Research, die zeigten, dass wissenschaftlich plausible Therapien häufig unwirksam und zuweilen sogar schädlich sein können, gewann eine pragmatisch orientierte klinische Forschung zunehmend an Boden, und die beherrschende Dominanz der Plausibilität wurde zumindest zum Teil gebrochen. Durch diese Entwicklungen, vor allem aber durch die enorm wachsende Inanspruchnahme komplementärer Verfahren [6], wurde Forschung zu komplementären Verfahren zumindest zu einer geduldeten Aktivität, wenn auch eine systematische Förderung in den meisten Ländern nach wie vor aussteht. Mit der zunehmenden Zahl hochwertiger, randomisierter klinischer Studien in der Komplementärmedizin wächst aber auch die Erkenntnis, dass ein überzeugender Wirksamkeitsnachweis im üblichen Sinne häufig nicht oder nur schwer zu erbringen ist. Klinische Studien haben zahllose Fehlerquellen, und die Ergebnisse sind häufig widersprüchlich. Die Evidenzlage bezüglich placebokontrollierter Studien ist besonders in der Homöopathie so verwirrend, dass weitere Einzelstudien und Metaanalysen die Debatte derzeit kaum weiterbringen können. Selbst bei vergleichsweise gut belegten Massnahmen wie z.B. dem Einsatz von Johanniskraut bei Depression sind die Belege nicht ganz eindeutig. Positive Ergebnisse aus einer hochwertigen Grundlagenforschung würden bei Verfahren wie der Homöopathie die Akzeptanzvoraussetzungen fundamental verbessern. Die bisherigen Ergebnisse und die allgegenwärtigen Probleme der Reproduzierbarkeit machen aber angesichts der fehlenden Ressourcen für eine echte Evaluationsstrategie nicht gerade Hoffnung, dass hier in nächster Zeit ein Durchbruch bevorstehen könnte. Negative Ergebnisse sind von vorneherein kaum interpretierbar, da die Relevanz von präklinischen Modellen für die Homöopathie unklar ist. Bei Phytotherapeutika wie Echinacea oder Johanniskraut ist die Beleglage in der präklinischen Forschung dagegen nicht so schlecht;

\begin{tabular}{ll}
\hline KARGER & $\oplus 2005$ S. Karger GmbH, Freiburg \\
Fax +49 7614520714 & Accessible online at: \\
$\begin{array}{l}\text { E-mail Information@Karger.de } \\
\text { www.karger.com }\end{array}$ & www.karger.com/fkm
\end{tabular}


trotzdem sind eindeutige Schlussfolgerungen schwierig. In dieser Situation fällt es nicht leicht zu begründen, warum wir für unsere komplementärmedizinische Forschung unbedingt mehr (bzw. überhaupt irgendwelche) öffentliche Gelder bekommen müssen.

Auf der anderen Seite mehren sich die Hinweise, dass komplementärmedizinische Verfahren in der Versorgung irgendwie «funktionieren». Diese Hinweise stammen meist aus pragmatischen randomisierten Studien und aus nichtrandomisierten Studien [z.B. 7, 8]. Wirklich überzeugend sind Qualität und Aussagekraft dieser Studien bisher zwar nur selten. Zusammen mit dem enormen Zuspruch durch die Patienten sprechen sie aber doch dafür, dass hier relevante, offensichtlich durch die «konventionelle» Medizin nicht bediente Bedürfnisse befriedigt werden. In ihrem Editorial fordern die Lancet-Herausgeber nicht nur, dass Ärzte ihren Patienten ehrlich sagen, dass Homöopathie nichts bringt, sondern auch, dass es der modernen Medizin nicht gelingt «to address the patients' needs for personalised care».

Interessanterweise bestreiten auch Skeptiker kaum, dass sogar unplausible komplementäre Verfahren in der Praxis zum Teil positive Auswirkungen haben können. Die Einschätzung, dass entsprechende Interventionen «besonders gute Placebos» seien, ist erstaunlich. Zum einen ist die Existenz klinisch relevanter Placeboeffekte insgesamt nicht ganz unumstritten $[9,10]$. Zum anderen hätte es methodisch fundamentale Folgen, wenn unterschiedliche Placebos unterschiedlich wirksam wären. Wenn z.B. eine «Placeboakupunktur» ein besseres Placebo wäre als ein medikamentöses Placebo, hätte es die Akupunktur nicht nur schwerer, ihre Überlegenheit zu beweisen; es würde auch bedeuten, dass es so etwas wie «spezifische Placeboeffekte» gibt. Die bisher nur zum Teil vorliegenden Ergebnisse aus den Modellvorhaben zur Akupunktur deuten in diese Richtung [11, 12].

Es stellt sich die Frage, ob die Schwerpunkte in der klinischen
Forschung zu komplementärmedizinischen Verfahren verschoben werden sollten. Dabei geht es nicht primär um die unendliche Designdebatte (randomisiert oder nicht). Die Mehrheit der Personen, die klinische Forschung zu komplementärmedizinischen Therapien betreiben, geht implizit davon aus, dass «ihr» Verfahren letztlich «spezifisch» wirksam ist: beim Homöopathen die Hochpotenzen, beim Akupunkteur die nach chinesischer Syndromdiagnose gewählten Punkte usw. Entsprechend werden die Fragen gestellt und die Methoden gewählt - auch in unkontrollierten Studien. Wir sollten uns viel ernsthafter mit der Möglichkeit auseinander setzen, dass ein relevanter Teil der klinisch beobachtbaren Effekte nicht auf den jeweils postulierten (oft wissenschaftlich wenig plausiblen bis unplausiblen) Wirkungsmechanismus zurückzuführen ist, dennoch aber kein «unspezifischer Placeboeffekt» im üblichen Sinne ist. Dementsprechend muss viel stärker als bisher der Einfluss von Einstellungen, Erwartungen, Bedeutungen, behandlungsassoziierten Handlungs- und Interaktionsmustern usw. mituntersucht werden [13]. Das heisst nicht, dass komplementärmedizinische Interventionen grundsätzlich keine spezifischen Wirkungen haben. Aber wir sollten auf die häufig ernüchternden Ergebnisse vieler guter klinischer Studien nicht nur dadurch reagieren, mit anderen Designs letztlich dass Gleiche zu untersuchen.

Editorials und Ergebnisse wie jetzt im New England Journal of Medicine oder im Lancet dürften die ohnehin schon geringe Bereitschaft, Geld für komplementärmedizinische Forschung auszugeben, weiter vermindern. Ein bewusster und expliziterer Einbezug von Elementen einer Art Placeboforschung könnte die Chancen von Forschungsanträgen aber verbessern. Zumindest dem Autor dieses Editorials scheint dies nicht nur ein Argument, um Förderer davon zu überzeugen, dass ihr Geld in der komplementärmedizinischen Forschung nicht verschwendet ist, sondern auch ein Weg, um unsere begrenzten Ressourcen besser zu nutzen.

\section{Literatur}

1 Sampson W: Studying herbal remedies. N Engl J Med 2005;353:337-9.

2 Turner RB, Bauer R, Woelkart K, Hulsey TC, Gangemi JD: An evaluation of Echinacea angustifolia in experimental rhinovirus infections. $\mathrm{N}$ Engl $\mathrm{J}$ Med 2005;353:341-8.

3 Shang A, Huwiler-Müntener K, Nartey L, Jüni P, Dörig S, Sterne JAC et al: Are the clinical effects of homoeopathy placebo effects? Comparative study of placebo-controlled trials of homoeopathy and allopathy. Lancet 2005;366:726-32.

4 Linde K, Clausius N, Ramirez G, Melchart D, Eitel F, Hedges LV et al: Are the clinical effects of homoeopathy placebo effects? A meta-analysis of placebo-controlled trials. Lancet 1997;350:834-43.

5 The Lancet: The end of homoeopathy. Lancet 2005;366:690.
6 Härtel U, Volger E: Inanspruchnahme und Akzeptanz klassischer Naturheilverfahren und alternativer Heilmethoden in Deutschland - Ergebnisse einer repräsentativen Bevölkerungsstudie. Forsch Komplementärmed Klass Naturheilkd 2004;11: 327-334.

7 Vickers AJ, Rees RW, Zollman CE, McCarney R, Smith C, Ellis N et al: Acupuncture for chronic headache in primary care: large, pragmatic, randomised trial. BMJ 2004;328:744-7.

8 Hamre HJ, Fischer M, Heger M, Riley D, Haidvogl M, Baars E et al: Anthroposophic vs. conventional therapy of acute respiratory and ear infections: a prospective outcomes study. Wien Klin Wochenschr 2005;117:256-68

9 Kienle G, Kiene H: Placeboeffekt und Placebokonzept - eine kritische methodologische und konzeptionelle Analyse von Angaben zum Ausmass des Placeboeffekts. Forsch Komplementärmed 1996;3: 121-38.
10 Hrobjartsson A, Gøtzsche PC: Is the placebo powerless? An analysis of clinical trials comparing placebo with no treatment. N Engl J Med 2001;344: 1594-602.

11 Hackenbroch V: Die eingebildete Heilung. Der Spiegel 2004(44):196-8.

12 Linde K, Streng A, Jürgens S, Hoppe A, Brinkhaus B, Witt $\mathrm{C}$ et al: Acupuncture for patients with migraine. A randomized controlled trial. JAMA. 2005;293:2118-25.

13 Giesser I, Wittmann M, Mitzdorf U: Behandlungsbezogene Einstellungen und Behandlungsmotivation. Forsch Kompementärmed Klass Naturheilkd DOI: $10.1159 / 000088290$ 